\title{
Model for thermal conductivity in nanoporous silicon from atomistic simulations
}

\author{
Riccardo Dettori, ${ }^{1}$ Claudio Melis, ${ }^{1}$ Xavier Cartoixà, ${ }^{2}$ Riccardo Rurali, ${ }^{3}$ and Luciano Colombo ${ }^{1, *}$ \\ ${ }^{1}$ Dipartimento di Fisica, Università di Cagliari, Cittadella Universitaria, I-09042 Monserrato (Ca), Italy \\ ${ }^{2}$ Departament d'Enginyeria Electrònica, Universitat Autònoma de Barcelona, 08193 Bellaterra, Barcelona, Spain \\ ${ }^{3}$ Institut de Ciència de Materials de Barcelona (ICMAB-CSIC) Campus de Bellaterra, 08193 Bellaterra, Barcelona, Spain
}

(Received 7 August 2014; revised manuscript received 24 November 2014; published 23 February 2015)

\begin{abstract}
By means of molecular dynamics simulations, we have studied heat transport in nanoporous silicon, finding that the Eucken model, widely adopted in the description of thermal transport in macroporous systems, breaks down when pores are nanometer-sized. Present atomistic results are used to inform an extension to this model, effectively describing the relationship between thermal conductivity and interface density, here identified as the key structural characteristic of a porous sample. Our model, validated against a range of pore sizes and distributions, provides a robust framework for the interpretation of the atomistic results, as well as suggesting how to estimate the average pore size through thermal transport measurements.
\end{abstract}

DOI: 10.1103/PhysRevB.91.054305

PACS number(s): 66.25.+g, 81.05.Rm, 44.30.+v

\section{INTRODUCTION}

Among many other intriguing properties, porous $\mathrm{Si}$ (PS) is characterized by a thermal conductivity $\kappa$ up to three orders of magnitude smaller than in crystalline $\mathrm{Si}$ (c-Si) [1] and by the ease of increasing the concentration of charge carriers by gas adsorption $[2,3]$. These features make it very promising for thermoelectric conversion. PS is also a perfect candidate for thermal insulation [4,5] and, therefore, is under extensive investigation as a key energy material.

Several experimental studies have been performed, reporting a wide range of $\kappa$ values, depending either on doping and on fabrication techniques [6]. The lowest values $0.04 \leqslant$ $\kappa \leqslant 1.2 \mathrm{~W} \mathrm{~m}^{-1} \mathrm{~K}^{-1}$ have been achieved for $p$ - and $p^{+}$-doped silicon in disordered porous samples with porosity varying from $40 \%$ to $80 \%$ [7-9]. Tang et al. [10] instead focused on the thermoelectric properties of samples with cylindrical pores arranged in a hexagonal pattern, reporting that $\kappa$ is reduced by a factor 100 with respect to $\mathrm{c}-\mathrm{Si}$, reaching a figure of merit of $\mathrm{ZT} \sim 0.4$. From a theoretical point of view, Lee et al. [5,11] used a combination of classical molecular dynamics (MD) and $a b$ initio density functional theory to study the thermoelectric properties of nano-PS, characterized by periodically arranged circular and square pores, estimating $0.6 \leqslant \kappa \leqslant 2.5 \mathrm{~W} \mathrm{~m}^{-1} \mathrm{~K}^{-1}$, and $\mathrm{ZT}=0.4$. He et al. [1] performed MD and lattice dynamics calculations in thin films with cylindrical pores, showing that $\kappa$ could be reduced up to a factor 20 with respect to bulk c-Si.

Although the above scenario offers quite a number of reliable determinations of $\kappa$ in many PS samples differing by morphology, a general picture is still missing in correlating the heat transport properties to some overall feature related to porosity. This is detrimental to understanding possible general trends, as we indeed aim at in the present work. In particular, comparatively little attention has been so far given to characterize by atomistic simulations thermal transport in samples with disordered porosity, a configuration closer to the experimental situation. As a matter of fact, it is very unlikely to obtain a real sample with an ordered array of pores especially

*1uciano.colombo@dsf.unica.it when using etching fabrication processes which do not allow to control the spatial and shape distribution of voids. This work is addressed to fill this gap: atomistic simulations are used to inform an effective model describing on a general basis the relationship between $\kappa$ and interface density (i.e., the ratio between porosity and average pore diameter). The model here developed, including and extending the previous Eucken model valid only for macroporous composites [12,13], is applied to both ordered and random nano-PS structures, providing a robust rationale to understand and predict $\kappa$ values, once the system morphology has been given.

\section{BACKGROUND}

\section{A. Computational details}

Thermal conductivity has been calculated in periodically repeated simulation cells during the system approach to equilibrium (occurred in a microcanonical MD run), proceeding from an initial nonequilibrium configuration where the left and right halves of the simulation cell were set at temperature $T_{1}=400 \mathrm{~K}$ and $T_{2}=200 \mathrm{~K}$, respectively. By computing on-the-fly the evolving temperature difference $\Delta T(t)=\left\langle T_{1}(t)\right\rangle-\left\langle T_{2}(t)\right\rangle$ (where $\langle\cdots\rangle$ indicates the average separately taken in the two half-cells), we straightforwardly determined $\kappa$ from

$$
\Delta T(t)=\left\langle T_{1}\right\rangle-\left\langle T_{2}\right\rangle=\sum_{n=1}^{\infty} C_{n} e^{-\alpha_{n}^{2} t \kappa / \rho \mathcal{C}_{v}},
$$

where $\rho$ is the mass density and $\mathcal{C}_{v}$ is the specific heat of the simulated system. Equation (1) is obtained by solving exactly the heat equation, as extensively discussed elsewhere [16]. The coefficients

$$
C_{n}=8\left(T_{1}-T_{2}\right) \frac{\left[\cos \left(\alpha_{n} L_{z} / 2\right)-1\right]^{2}}{\alpha_{n}^{2} L_{z}^{2}}
$$

and $\alpha_{n}=2 \pi n / L_{z}$ are obtained for a system of length $L_{z}$ initially prepared in the configuration described above. Typically, Eq. (1) is developed up to the $n=20$ term. This method, hereafter referred to as approach to equilibrium molecular dynamics (AEMD), has been successfully applied 
to investigate thermal transport properties in nanostructured Si-based systems [14-18].

The equations of motion have been integrated by the velocity-Verlet algorithm with a time step as short as 2 fs and simulations have been performed using the LAMMPS [19] package and the environment dependent interatomic potential (EDIP) [20]. This interaction scheme has been adopted since it is accurate in describing noncrystalline forms of silicon, as indeed required for the present investigations. More importantly, it has been elsewhere established its accuracy in predicting the thermal transport properties of disordered silicon forms, like, e.g., amorphous and nanocrystalline ones [16]. Finally, the reliability of the present simulation protocol is also proved by the good agreement found for the AEMD thermal conductivity of $\mathrm{c}-\mathrm{Si}$ with the Boltzmann transport equation prediction of Refs. [21-23] based on the same EDIP potential, although the actual value (i.e., $\kappa=294 \mathrm{~W} \mathrm{~m}^{-1} \mathrm{~K}^{-1}$ ) is somewhat larger than the experimental one.

\section{B. Sample generation}

The bulk c-Si matrix has been modeled by a simulation cell with length $L_{z}$ varying in the range $24 \mathrm{~nm} \leqslant L_{z} \leqslant 136 \mathrm{~nm}$ (see below) and fixed $13.6 \times 13.6 \mathrm{~nm}^{2}$ section in the $x y$ plane. The resulting number of atoms varied therefore from $0.2 \times 10^{6}$ to $1.3 \times 10^{6}$, corresponding to simulation cells large enough to reproduce all the main structural features of PS. We studied two different kinds of system: ordered porous samples (OPS), where the porosity is created by arranging a periodical array of spherical pores, and random porous samples (RPS), with randomly distributed, sized and shaped pores.

In general, the porosity

$$
\varphi \equiv \frac{V_{\text {pores }}}{V_{\text {system }}}
$$

is defined as the ratio between the total volume $V_{\text {pores }}$ of the regions where $\mathrm{Si}$ atoms have been removed and the system volume $V_{\text {system }}$ [24]. The porosity itself does not provide any relevant information on the system morphology since, at least for OPS, it depends on two parameters: the number of the pores $N_{p}$, and the pore diameter $d_{p}$, i.e., $\varphi=\varphi\left(N_{p}, d_{p}\right)$. For example, the same given porosity $\varphi$ can be obtained by creating a large number of small pores or by just few larger ones. Three examples of OPS are shown in Fig. 1, characterized by the same porosity $\varphi=0.14$, but different pore size $d_{p}$. As we will explain in the next section, the quantity that identifies accurately the porous system and its inner surfaces is the interface density $\Psi$. In particular, we will show that the overall thermal conductivity monotonically depends on $\Psi$.

As for OPS, spherical pores with diameter $d_{p}$ were arranged in a simple cubic lattice or in a thetragonal lattice: the array of pores was created by assigning the positions of the center of the pores and then by removing the atoms contained in the sphere with radius $d_{p} / 2$ (see Fig. 1).

As for RPS, we added educated guesses to the above procedure in order to enforce a twofold character of the resulting spatial distribution of pores, namely: randomness and uniformness. The uniform distribution of pores is not only intended to mimic a typical experimental condition [6], but it is also required by the assumption underlying the AEMD

$$
d=1.8 \mathrm{~nm}-\varphi=0.14
$$

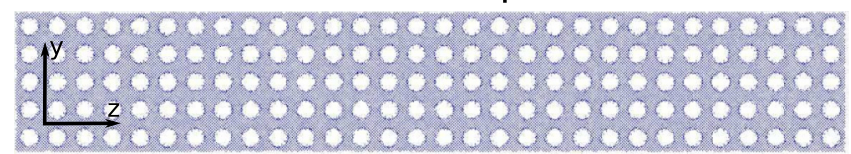

$$
d=3.3 \mathrm{~nm}-\varphi=0.14
$$

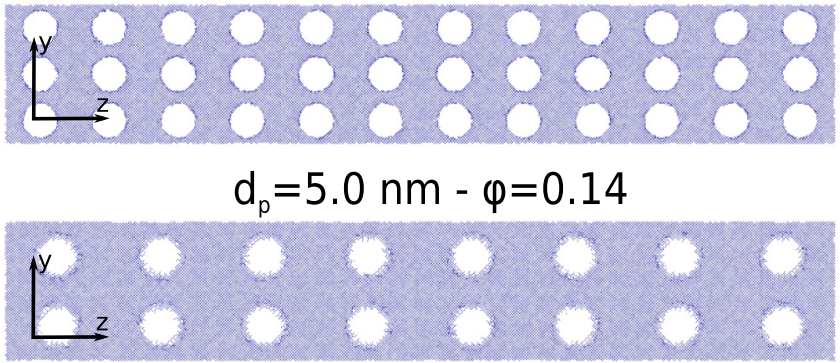

FIG. 1. (Color online) Ordered porous Si samples with same porosity $\varphi=0.14$, but different pore diameter $d_{p}$. Pictures show a 1.36-nm-thick longitudinal section. Heat flux is generated along the $z$ direction (see text).

method [16]. We also carefully equilibrated the RPS structure by simulated annealing at $900 \mathrm{~K}$ temperature, followed by a further equilibration at room temperature for a total simulation time of $0.6 \mathrm{~ns}$. This procedure allows inner surfaces to fully relax towards a highly-defected structure, very similar to amorphous silicon. In Fig. 2, we report the calculated $\varphi$ along the $z$ direction of a typical RPS, showing that the deviation around the preset value of $\varphi=0.30$ is indeed very small.

Finally, we remark that when dealing with disordered structures like RPS (which are shown in Fig. 3) configurational averaging is requested in order to provide quantitative information. Therefore, for each value of porosity, we averaged the thermal conductivity over four different samples (with fixed size and porosity).

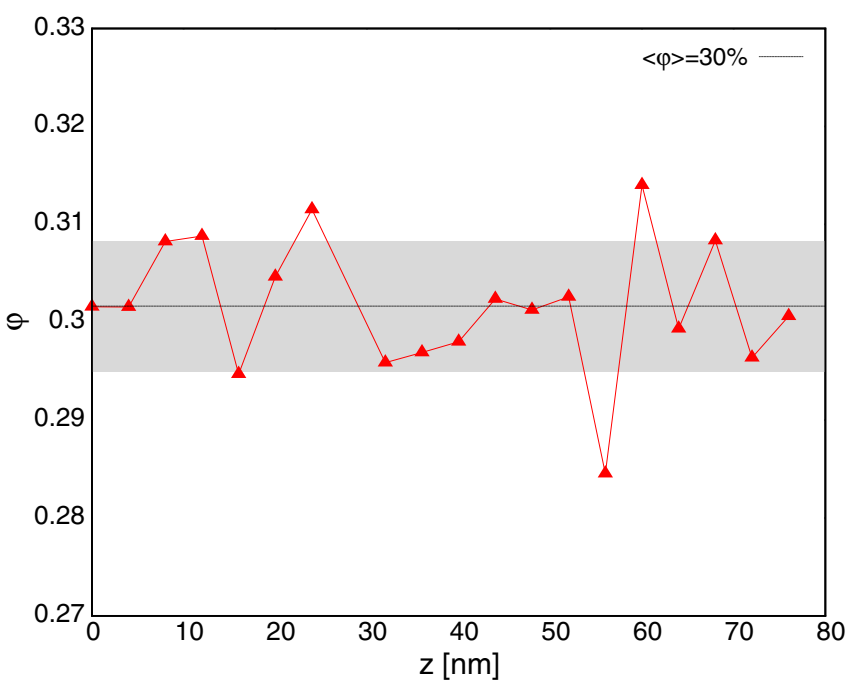

FIG. 2. (Color online) Red triangles: local porosity of a typical random porous $\mathrm{Si}$ sample calculated along the $z$ direction. Horizontal solid line: average porosity. Shaded area: standard deviation for porosity. 


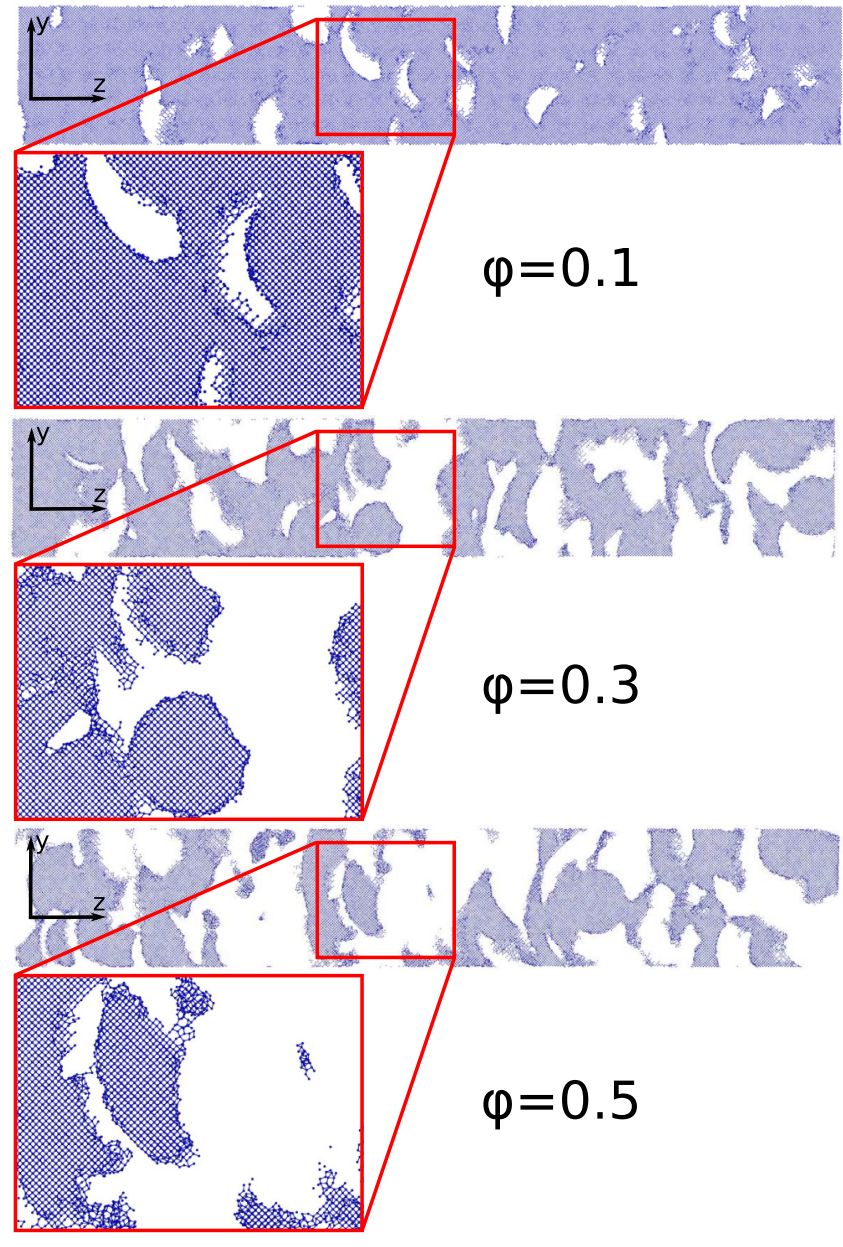

FIG. 3. (Color online) Random porous Si samples with different porosity $\varphi$. Pictures show a 1.36-nm-thick longitudinal section. The zoomed frames provide additional details on the atomic scale structure. We remark that the simulation cell is as thick as $13.6 \mathrm{~nm}$ and, therefore, matter is not discontinuous. Heat flux is generated along the $z$ direction (see text).

\section{Size effects}

It is well known that in MD simulations performed on cells with finite length $L_{z}$ it occurs that $\kappa=\kappa\left(L_{z}\right)$ due to a fraction of the heat carriers having a mean free path (MFP) longer than $L_{z}$ [21,25]. Therefore really very large cells (simulating the regime $L_{z} \rightarrow \infty$ ) should be required

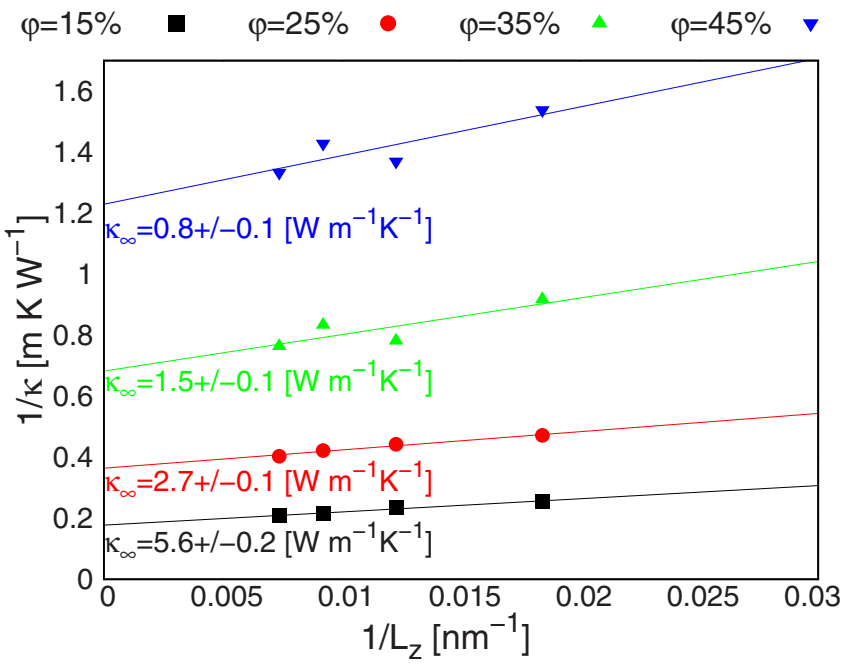

FIG. 4. (Color online) Inverse thermal conductivity $1 / \kappa$ vs inverse cell size $1 / L_{z}$ for ordered porous $\mathrm{Si}$ samples with different porosity. Symbols are typically as large as the standard deviation of the calculated thermal conductivity.

for a single-run reliable prediction. This is obviously not a valid approach, because of the overwhelming computational workload required. A more practical solution provided in Refs. [21,25] consists in performing a set of calculations for increasing (but still affordable) values of $L_{z}$. Then, by plotting $1 / \kappa$ versus $1 / L_{z}$, the thermal conductivity $\kappa_{\infty}$ for an ideally infinite sample is obtained by extrapolating the simulation data down to the $1 / L_{z} \rightarrow 0$ limit. This extrapolation procedure is good provided that one considers a set of $L_{z}$ values at least comparable with the maximum MFP of heat carriers in the investigated system.

By adopting this procedure, we investigated size effects in OPS by considering four values of porosity in the range $0.15 \leqslant \varphi \leqslant 0.45$ and by calculating $\kappa\left(L_{z}\right)$ for systems with $54.3 \mathrm{~nm} \leqslant L_{z} \leqslant 135.8 \mathrm{~nm}$. The results are shown in Fig. 4 and also reported numerically in Table I. A linear $1 / \kappa$ versus $1 / L_{z}$ trend is found, although its slope is comparatively much smaller than in c-Si [16], providing evidence that, for the system dimensions here considered, size only marginally affects thermal conductivity predictions. Consistently with previous findings $[1,26]$, this suggests that pores indeed act as very efficient scatterers for heat carriers, causing a considerable reduction of their mean free path.

TABLE I. Thermal conductivity $\kappa$ for various ordered porous Si samples, differing in porosity $\varphi$, pore diameter $d_{p}$, pore number $N_{p}$ and size $L_{z}$. These data are also reported in Fig. 4 .

\begin{tabular}{llcccc}
\hline \hline & \multicolumn{4}{c}{$\kappa\left(\mathrm{W} \mathrm{m}^{-1} \mathrm{~K}^{-1}\right)$} \\
\cline { 2 - 6 }$L_{z}(\mathrm{~nm}) N_{p}$ & $\varphi=0.15$ & $\varphi=0.25$ & $\varphi=0.35$ & $\varphi=0.45$ \\
\hline 54.399 & $d_{p}=3.0 \mathrm{~nm}$ & $d_{p}=3.5 \mathrm{~nm}$ & $d_{p}=3.9 \mathrm{~nm}$ & \\
81.5 & 153 & 3.91 & 2.12 & 1.09 & \\
108.6 & 4.26 & 2.26 & 1.28 & 0.65 \\
135.8252 & 4.67 & 2.37 & 1.20 & 0.73 \\
$\quad \infty$ & 4.77 & 2.48 & $1.5 \pm 0.1$ & $0.75 \pm 0.1$ \\
(extrapolated) & $5.6 \pm 0.2$ & $2.78 \pm 0.1$ & & \\
\hline
\end{tabular}


TABLE II. Thermal conductivity $\kappa$ for various random porous Si samples, differing in porosity $\varphi$ and size $L_{z}$. These data are also reported in Fig. 5. The indicated errors reflect a configurational average over 4 different samples. The average thermal conductivity $\langle\kappa\rangle$ is reported for any porosity.

\begin{tabular}{lllll}
\hline \hline & \multicolumn{4}{c}{$\kappa\left(\mathrm{W} \mathrm{m}^{-1} \mathrm{~K}^{-1}\right)$} \\
\cline { 2 - 5 }$L_{z}$ & $\varphi=0.15$ & $\varphi=0.25$ & $\varphi=0.35$ & $\varphi=0.45$ \\
\hline 24.4 & $3.2 \pm 0.1$ & $2.2 \pm 0.5$ & $0.8 \pm 0.2$ & $0.9 \pm 0.3$ \\
32.6 & $3.5 \pm 0.1$ & $2.0 \pm 0.5$ & $1.2 \pm 0.2$ & $0.8 \pm 0.3$ \\
40.7 & $4.8 \pm 0.1$ & $1.7 \pm 0.5$ & $0.8 \pm 0.2$ & $0.7 \pm 0.3$ \\
54.3 & $3.1 \pm 0.1$ & $2.0 \pm 0.5$ & $1.0 \pm 0.2$ & $0.4 \pm 0.3$ \\
81.5 & $5.8 \pm 0.1$ & $2.9 \pm 0.5$ & $0.9 \pm 0.2$ & $0.7 \pm 0.3$ \\
108.6 & $4.3 \pm 0.1$ & $1.6 \pm 0.5$ & $1.0 \pm 0.2$ & $0.4 \pm 0.3$ \\
135.8 & $4.6 \pm 0.1$ & $2.2 \pm 0.5$ & $1.2 \pm 0.2$ & $0.4 \pm 0.3$ \\
$\langle\kappa\rangle$ & $4.2 \pm 1.0$ & $2.1 \pm 0.5$ & $1.0 \pm 0.2$ & $0.6 \pm 0.3$ \\
\hline \hline
\end{tabular}

As for RPS, we explored a larger range of system sizes, corresponding to $24.4 \mathrm{~nm} \leqslant L_{z} \leqslant 135.8 \mathrm{~nm}$. The results are summarized in Table II: interestingly enough, we observe a really weak dependence of $\kappa$ on $L_{z}$ (even at very low porosity), as confirmed by Fig. 5. The conclusion is straightforward: a random distribution of unequally shaped and sized voids fully inhibits the long-range features of any vibrational modes, regardless the actual value of $\varphi$.

Based on the benchmark calculations described in this section, we argue that in nanoporous silicon the estimation of the thermal conductivity by AEMD is only marginally affected (OPS), or even not at all affected (RPS), by the selected length $L_{z}$ of the simulation cell. Therefore we limited the following investigations to just one reference system size, namely $L_{z}=81.6 \mathrm{~nm}$. This offers the best compromise between numerical convenience and accuracy (as shown in Figs. 4 and 5).

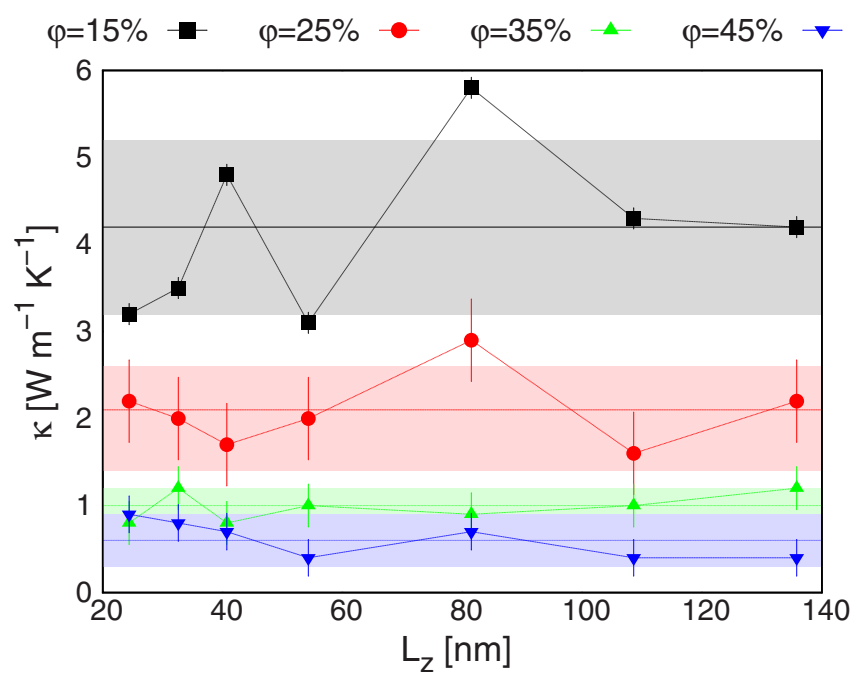

FIG. 5. (Color online) Thermal conductivity $\kappa$ as a function of cell size $L_{z}$ for random porous $\mathrm{Si}$ system with different porosity. The horizontal solid lines (shaded area) represent the average values (standard deviation).
TABLE III. Thermal conductivity $\kappa$ for various ordered porous Si samples with increasing porosity $\varphi$ and pore diameter $d_{p}$. The number of pores is fixed at $N_{p}=750$.

\begin{tabular}{lcc}
\hline \hline$\varphi$ & $d_{p}(\mathrm{~nm})$ & $\kappa\left(\mathrm{W} \mathrm{m}^{-1} \mathrm{~K}^{-1}\right)$ \\
\hline 0.03 & 1.0 & 8.67 \\
0.06 & 1.3 & 6.24 \\
0.09 & 1.6 & 4.93 \\
0.14 & 1.8 & 2.70 \\
0.22 & 2.0 & 1.71 \\
0.31 & 2.3 & 0.87 \\
0.34 & 2.4 & 0.73 \\
\hline \hline
\end{tabular}

\section{RESULTS AND DISCUSSION}

\section{A. $\kappa$ versus porosity: ordered systems}

As mentioned before, in OPS $\varphi$ only depends on $N_{p}$ and $d_{p}$. Therefore we can vary the porosity either by fixing the number of pores and by varying their diameter or, conversely, by distributing a different number of equally sized pores.

The first option was exploited by generating seven different samples with $0.03 \leqslant \varphi \leqslant 0.34$, each containing as many as $N_{p}=750$ identical pores (with suitable dimension to accomodate any assigned porosity) arranged in a simple cubic lattice. The second option, instead, was exploited by generating a new set of seven different samples, where an increasing number of pores was placed on a thetragonal lattice so to span the $0.02 \leqslant \varphi \leqslant 0.27$ porosity range. In this case, all pores have the same dimension $d_{p}=3.3 \mathrm{~nm}$. For this second case study, the procedure was also repeated by choosing $d_{p}=$ $5.0 \mathrm{~nm}$. The results for the two procedures are summarized, respectively, in Table III and in Tables IV and V. They compare reasonably well with Ref. [10] where (despite the fact that pores, arranged in an hexagonal regular pattern, have a much larger average dimension ranging from 55 to $350 \mathrm{~nm}$ ) samples with $\varphi=0.35$ are characterized by a thermal conductivity in the range $1.73 \mathrm{~W} \mathrm{~m}^{-1} \mathrm{~K}^{-1} \leqslant \kappa \leqslant 10.23 \mathrm{~W} \mathrm{~m}^{-1} \mathrm{~K}^{-1}$.

The common feature is that the thermal conductivity of OPS is greatly reduced with respect to the bulk crystalline value. However, samples obtained by following the first procedure have, on average, a much smaller $\kappa$, precisely: as small as $50 \%$ or $30 \%$ of the corresponding one for samples obtained by the second procedure with $d_{p}=3.3$ or $5.0 \mathrm{~nm}$, respectively. Although these results are expected and qualitatively explained by the presence of several internal

TABLE IV. Thermal conductivity $\kappa$ for various ordered porous Si samples with increasing porosity $\varphi$ and number of pores $N_{p}$. The pore diameter is fixed at $d_{p}=3.3 \mathrm{~nm}$.

\begin{tabular}{lrc}
\hline \hline$\varphi$ & $N_{p}$ & $\kappa\left(\mathrm{W} \mathrm{m}^{-1} \mathrm{~K}^{-1}\right)$ \\
\hline 0.02 & 18 & 22.22 \\
0.05 & 36 & 11.01 \\
0.09 & 72 & 6.15 \\
0.14 & 108 & 4.61 \\
0.18 & 144 & 3.75 \\
0.23 & 180 & 3.22 \\
0.27 & 216 & 2.96 \\
\hline \hline
\end{tabular}


TABLE V. Thermal conductivity $\kappa$ for various ordered porous $\mathrm{Si}$ samples with increasing porosity $\varphi$ and number of pores $N_{p}$. The pore diameter is fixed at $d_{p}=5.0 \mathrm{~nm}$.

\begin{tabular}{lrc}
\hline \hline$\varphi$ & $N_{p}$ & $\kappa\left(\mathrm{W} \mathrm{m}^{-1} \mathrm{~K}^{-1}\right)$ \\
\hline 0.03 & 8 & 24.62 \\
0.05 & 12 & 12.17 \\
0.07 & 16 & 9.54 \\
0.12 & 28 & 7.06 \\
0.14 & 32 & 6.64 \\
0.17 & 40 & 5.98 \\
0.21 & 48 & 5.16 \\
\hline \hline
\end{tabular}

interfaces (at each matrix/pore boundary), which provide an efficient phonon scattering mechanism, a more quantitative picture is definitely needed in order to elaborate a rationale for the observed trends.

\section{B. Effective thermal conductivity in porous media}

There have been many attempts to provide a general law $\kappa=\kappa(\varphi)$. The most widely referenced model was originally proposed by Eucken, based on an effective medium approximation for two-phase porous media [12,13,27-29]. There, the first and second phases are, respectively, the material forming the host matrix and the embedded voids. By assuming that the second phase is organized in spheres and that the distance between the spheres is much larger than $d_{p}$, the Eucken model leads to the relation

$$
\kappa_{\text {eff }}(\varphi)=\kappa_{\text {bulk }} \frac{1-\varphi}{1+\frac{\varphi}{2}}
$$

that accounts for the reduction of the thermal conductivity $\kappa_{\text {bulk }}$ of the first-phase material, caused by the presence of the pores. Equation (4) accurately describes the thermal conduction properties of composites with macropores, i.e., when the MFP of the vibrational modes of the pristine (first phase) material is smaller than the typical pore size. Conversely, in nanoporous materials (like those ones addressed in the present investigation) the situation is just the opposite. Furthermore, Eq. (4) is basically scale-invariant, as typical of effective medium theories. Therefore it does not carry any information about the dependence of thermal conduction on structural details like, e.g., the pore size (as indeed reported experimentally [30,31]). Any model aimed at improving the Eucken one must fully exploit the above two features, namely: the actual presence of pores (common to both macro- and nanoporous systems) and their nanoscale features (which additionally affect, through atomic-scale details).

As for the first issue, we adopt the standard picture that the diffusion of microscopic heat carriers is affected by pores as well as by their mutual scattering. A different maximum mean free path for the carriers is therefore associated to each scattering mechanism, respectively: $\Lambda_{\text {pores }}$ and $\Lambda_{\text {bulk }}$. While for systems obeying Eq. (4) it is easily found that $1 / \Lambda_{\text {pores }}=2 \Lambda_{\text {bulk }} / \varphi$, the evaluation of $\Lambda_{\text {bulk }}$ requires an anharmonic lattice dynamics calculation. This second scattering mechanism is the dominant one in the pristine material (i.e., in the first phase matrix with no voids). Applying Matthiesen rule,

$$
\frac{1}{\Lambda_{\text {eff }}}=\frac{1}{\Lambda_{\text {bulk }}}+\frac{1}{\Lambda_{\text {pores }}},
$$

we straightforwardly obtain the effective distance $\Lambda_{\text {eff }}$ traveled by heat carriers when both the above mechanisms limiting their diffusion are properly taken into account.

As for nanoscale features, it is convenient to consider the interface density $\Psi$ easily defined as

$$
\Psi=\frac{N_{\text {pores }}}{V_{\text {system }}} 4 \pi\left(d_{p} / 2\right)^{2}=\frac{6 \varphi}{d_{p}} .
$$

A new scattering length $\Lambda_{\text {interface }}$ (associated to interfacespecific scattering events) is accordingly defined, that can be written in the following form:

$$
\Lambda_{\text {interfaces }}=\frac{1}{N_{\text {coll }}}=\frac{4}{\Psi}=\frac{2 d_{p}}{3 \varphi},
$$

where

$$
N_{\text {coll }}=\frac{\pi d_{p}^{2}}{4} \frac{N_{\text {pores }}}{V_{\text {system }}}
$$

is the number of interface scattering events per unit length. We remark that the factor $\pi d_{p}^{2} / 4$ is the cross section for scattering at spherical pore of diameter $d_{p}$. By adding this new scattering contribution to Eq. (5), we eventually get for a nanoporous system

$$
\Lambda_{\mathrm{eff}}=\left(\frac{1}{\Lambda_{\mathrm{bulk}}}+\frac{1}{\Lambda_{\mathrm{bulk}}} \frac{\varphi}{2}+\frac{\Psi}{4}\right)^{-1}=\frac{\Lambda_{\mathrm{bulk}}}{1+\frac{\varphi}{2}+\frac{3 \Lambda_{\mathrm{bulk}}}{2 d_{p}} \varphi} .
$$

In order to proceed further, we remark that the thermal conductivity of a homogeneous (i.e., containing no voids) material with heat capacity $\mathcal{C}_{v}$ is usually approximated as

$$
\kappa \sim \frac{1}{3} \mathcal{C}_{v} v_{g} \Lambda,
$$

where only the dominant heat carrier with MFP equal to $\Lambda$ and propagating with speed $v_{g}$ is considered. When considering a (nano)porous material, the heat capacity can be usefully replaced with an effective value $\mathcal{C}_{v \text {,eff }}=(1-\varphi) \mathcal{C}_{v \text {, bulk }}$ (where $\mathcal{C}_{v \text {,bulk }}$ is the specific heat of the first-phase material), since the heat capacity of the pores is negligible [32]. Furthermore, the phonon group velocity is mostly determined by shortrange interactions and we can assume that is not that much affected by the presence of the pores; this is confirmed by MD simulations and lattice dynamics calculations [1]. In conclusion, under these assumptions and by combining Eqs. (9) and (10), we eventually get

$$
\kappa_{\text {eff }}(\varphi)=\kappa_{\text {bulk }} \frac{1-\varphi}{1+\frac{\varphi}{2}+\frac{3 \Lambda_{\text {bulk }}}{2 d_{p}} \varphi},
$$

which provides a simple, but very informative general expression for the effective thermal conductivity in a nanoporous material. Interestingly enough, Eq. (11) not only contains information about the pristine matrix (through $\kappa_{\text {bulk }}$ and $\Lambda_{\text {bulk }}$ terms) and the overall porosity $\varphi$, but it also properly takes into account the actual morphology of the pores through the $d_{p}$ parameter. This feature represents a major step forward since it makes Eq. (11) able to describe nanoporous systems, 


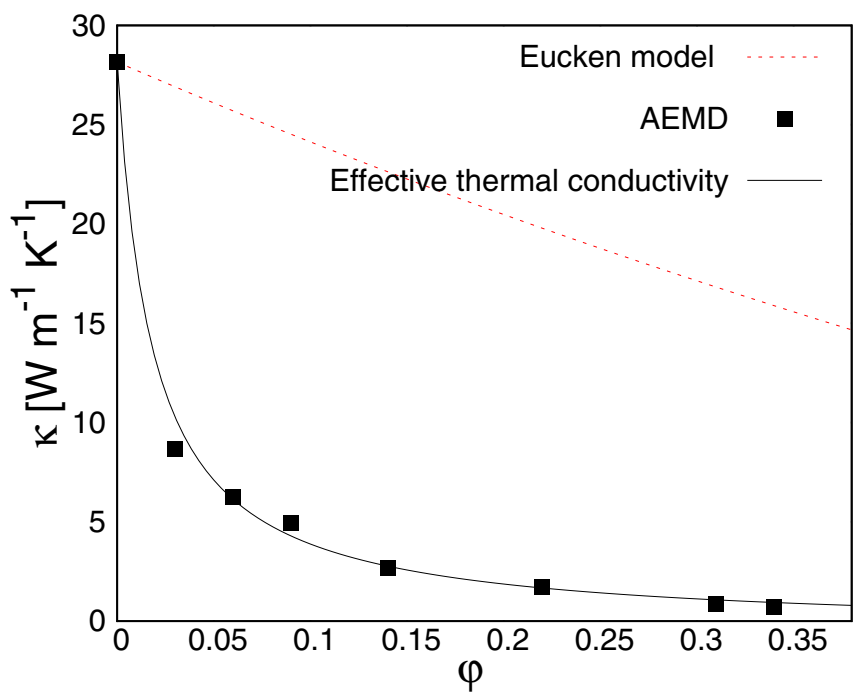

FIG. 6. (Color online) Thermal conductivity $\kappa$ as a function of porosity $\varphi$ in ordered porous $\mathrm{Si}$ samples with fixed number of pores. Black solid line: effective model provided by Eq. (11). Red dashed line: standard Eucken model provided by Eq. (4). Dots are typically as large as the standard deviation on $\kappa_{\text {eff }}$. Symbols are typically as large as the standard deviation of the calculated thermal conductivity.

at variance with Eq. (4), which is limited to macroporous composites. Nevertheless, the functional form of $\kappa_{\text {eff }}(\varphi)$ is the same of the Eucken model: in fact it is worth noting that for $d_{p} \gg \Lambda_{\text {bulk }}$ it reduces back to the Eucken model. The factor $\beta=\frac{3 \Lambda_{\text {bulk }}}{2 d_{p}}$ can be enclosed in a single parameter to be fit on the results of AEMD simulations.

In Fig. 6, we report the $\kappa$ values obtained by AEMD (black squares) for OPS with fixed pores; the solid black curve represents the effective thermal conductivity relation given in Eq. (11) that has been used to fit the data and the red dashed curve is the Eucken model. Fig. 6 clearly stands for the major improvement of the present model to the conventional Eucken one. The value for the $\beta$ parameter obtained by fitting the AEMD data is $55.3 \pm 4.4$. As $d_{p}$ is known for each point in the plot, we can use $\beta$ to calculate the values for $\Lambda_{\text {bulk }}$. This order-of-magnitude estimate predicts $40 \lesssim \Lambda_{\text {bulk }} \lesssim 90 \mathrm{~nm}$, in nice agreement with Ref. [21] where the dominant contribution to thermal conductivity in $\mathrm{c}-\mathrm{Si}$ is calculated to come from phonons with MFP as long as $\sim 10^{2} \mathrm{~nm}$.

In Fig. 7, we report a comparison between the two different classes of OPS here investigated, namely, those with fixed $N_{p}$ (black symbols) and those with fixed $d_{p}$ (red and green samples). For both $d_{p}=3.3$ and $5.5 \mathrm{~nm}$, the agreement between calculated AEMD data and Eq. (11) is definitely less good than by keeping $N_{p}$ fixed. This can be explained considering the local morphology of the samples: the structural relaxations at the pore/matrix interface result in the formation of an amorphous or highly-defected spherical shell surrounding the pore. The amount of defected matter increases with growing pore diameter, as shown in Fig. 8.

We remark once again that the Eucken model for $\kappa_{\text {eff }}(\varphi)$ has been derived by assuming a two-phase composite structure, while the observed defected/amorphous shell is, as far as the propagation of heat carries is concerned, effectively a third

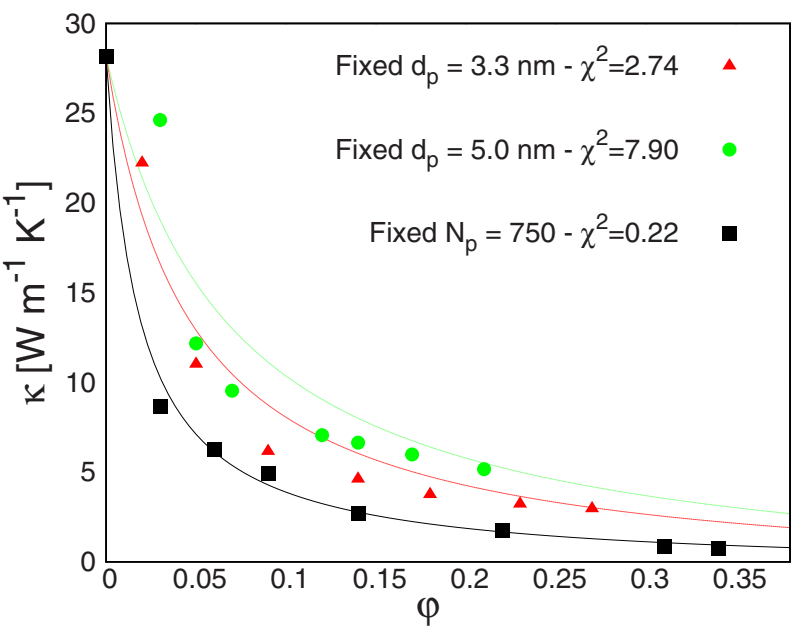

FIG. 7. (Color online) Thermal conductivity $\kappa$ as a function of porosity $\varphi$ in ordered porous Si samples with either fixed number of pores $N_{p}$ (black squares) and fixed pore diameter $d_{p}=3.3 \mathrm{~nm}$ (red triangles) and $d_{p}=5.0 \mathrm{~nm}$ (green dots). Solid lines: effective model provided by Eq. (11). Symbols are typically as large as the standard deviation of the calculated thermal conductivity.

phase: in the case of OPS with fixed $N_{p}$, the agreement is good because the pores are very small and there is little defected medium, while in the case of OPS with fixed $d_{p}$ (both values) the actual amount of a third-phase material is too large to be neglected. This statement is validated by counting the number (per pore) of atoms forming the third phase, as shown in Fig. 9. Defect atoms have been identified by evaluating their on-site energy, as calculated by the adopted EDIP potential: while in an ideal c-Si lattice at room temperature atoms have a configurational energy of about $\sim-4.6 \mathrm{eV} /$ atom, in the highly defected shells this value is raised above $-4.3 \mathrm{eV} /$ atom. Figure 9 clearly indicates that high-energy atoms have a much smaller occurrence in OPS with fixed $N_{p}$ : in this case Eq. (11) works at its best.

Another interesting feature of Fig. 7 worth of further investigation is that the larger are the pores, the worst is the agreement between calculated AEMD data and the model provided by Eq. (11), as indicated by the reduced $\chi^{2}$ of the fit reported in figure. Since $\kappa \sim\left(3 \Lambda_{\text {bulk }} / 2 d_{p}\right)^{-1}$ it is obvious that, for fixed porosity, the smaller are the pores the smaller is the thermal conductivity. This is also related to the interface density $\Psi=6 \varphi / d_{p}$ : for a given value of porosity it increases with decreasing pore diameter. In order to better point out this issue, in Fig. 10, we report the thermal conductivity of six OPS with same porosity $\varphi=0.28$, but different pore number and dimension. The corresponding values are listed in Table VI. The main conclusion is that, although the porosity in these system is constant, thermal transport is highly affected by an increasing interface density to which the probability of scattering is directly linked.

\section{C. $\kappa$ versus porosity: random systems}

In RPS it is hard to define the position, size, and shape of each pore. Therefore we rather characterize their nanoporous structure by providing the distribution of the pore dimensions 

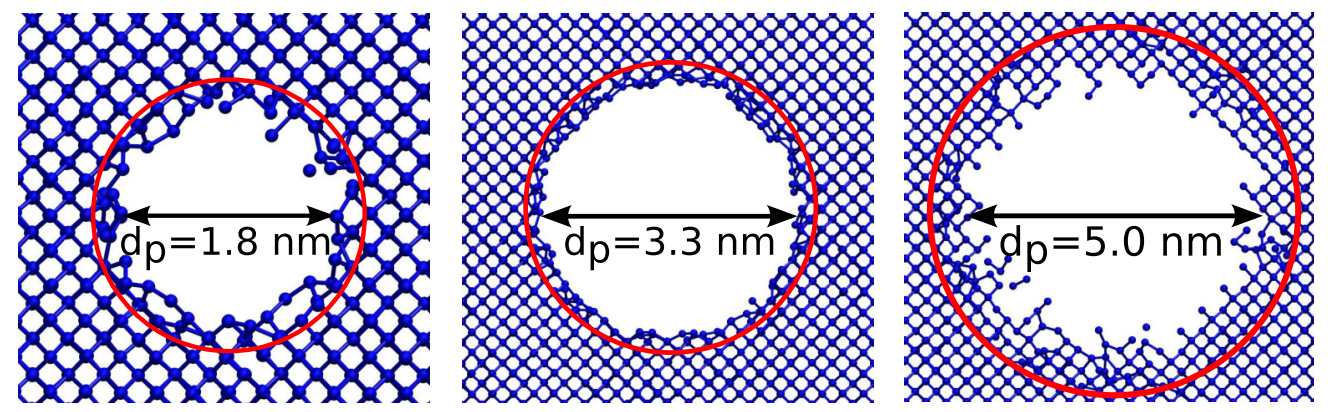

FIG. 8. (Color online) Detail of the microscopic structure nearby a typical spherical pore with increasing diameter $d_{p}$. The yellow circle identifies approximately the shell corresponding to the highly-defected region.

which, by construction, we set as a Gaussian distribution with average value $\left\langle d_{p}\right\rangle=4.0 \mathrm{~nm}$ and a standard deviation of $1.5 \mathrm{~nm}$. Table VII lists the results for several RPS with $0.05 \leqslant \varphi \leqslant 0.50$, showing, similarly to OPS, a monotonic decrease in thermal conductivity by increasing porosity. A direct comparison to experimental data is very hard, since little information is available for samples characterized at the nanoscale by a pore distribution similar to the one here investigated. However, in Ref. [33], the thermal conductivity in PS crystallites has been measured by the micro-Raman technique reporting $\kappa$ values well below $5 \mathrm{~W} \mathrm{~m} \mathrm{~m}^{-1} \mathrm{~K}^{-1}$ for any $\varphi \geqslant 0.3$. This is a further convincing argument supporting the results here presented. We also remark that the $\kappa=\kappa(\varphi)$ trend there reported is very similar to our findings. Furthermore, in Refs. [7,9], it is reported a thermal conductivity for high-porosity samples lower than 2 and $1.5 \mathrm{~W} \mathrm{~m}^{-1} \mathrm{~K}^{-1}$, respectively. Once again these results are in good agreement with those ones reported in Table VII. Finally, in Ref. [35], a direct measurement of thermal conductivity in $p^{+}$-doped

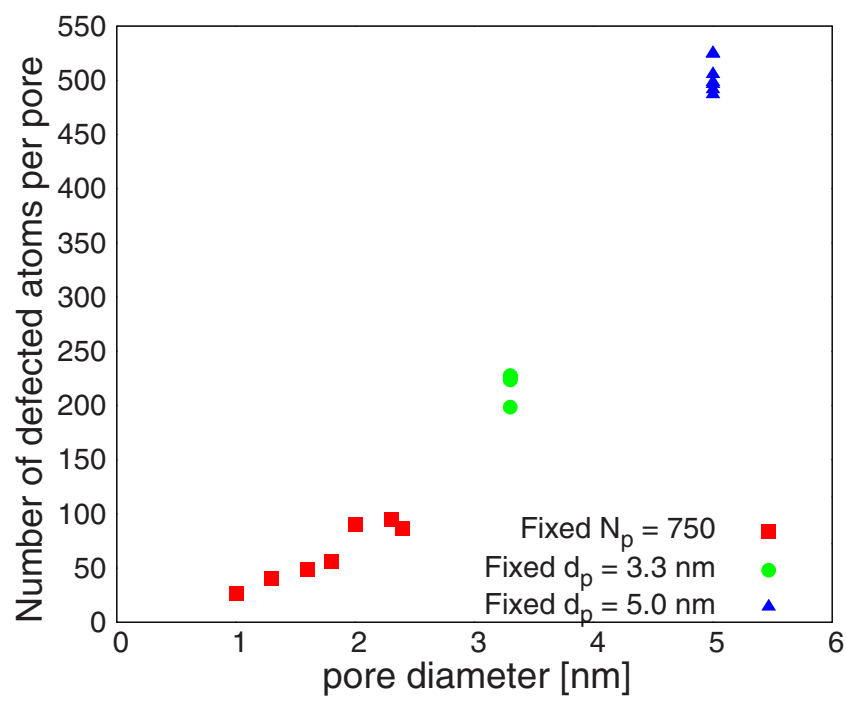

FIG. 9. (Color online) Number of defected atoms (per pore) as a function of the pore diameter in ordered porous Si samples. Red squares: systems with constant number of pores. Green dots (blue triangles): systems with pore diameter fixed at $d_{p}=3.3 \mathrm{~nm}\left(d_{p}=\right.$ $5.0 \mathrm{~nm})$. mesoporous silicon is reported with $\varphi \sim 0.5$ as small as $1 \mathrm{~W} \mathrm{~m}^{-1} \mathrm{~K}^{-1}$ while, for a similar porosity, the rather different value of $3.9 \mathrm{~W} \mathrm{~m}^{-1} \mathrm{~K}^{-1}$ is reported in Ref. [36], which further indicates the large scattering of experimental data.

It is remarkable that, as shown in Fig. 11, the thermal conductivity values in RPS are slightly larger than in OPS with fixed $N_{p}$, indicating a reduced interface density. This is due to the fact that, since pores do not overlap, the interface density is maximum in OPS. Furthermore, AEMD results for RPS are in good agreement with Eq. (11). This suggests that, for samples containing a random array of pores, $\kappa$ is neither affected by their actual shape, nor by their spatial distribution. As a further sanity check for our calculations, we evaluated the average pore diameter in RPS from the corresponding $\beta$ parameter. The result is $\left\langle d_{p}\right\rangle=4.3 \pm 1.3 \mathrm{~nm}$, fully consistent with the values used during the sample generation. Incidentally, this results suggests a possible practical procedure for estimating the pore average size, indeed a relevant information hard to get through thermal transport measurements [34].

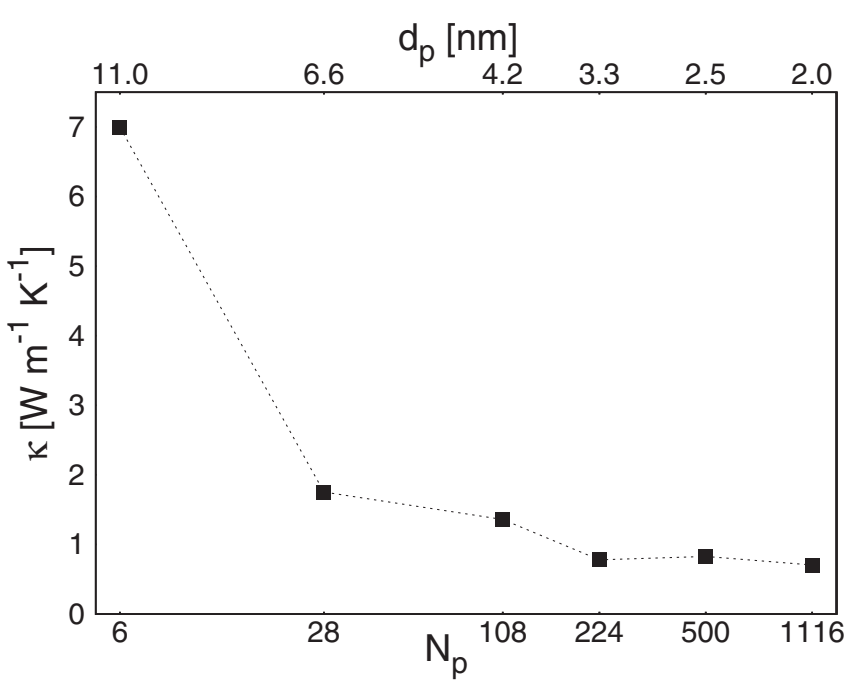

FIG. 10. Thermal conductivity $\kappa$ as a function of the number of pores $N_{p}$ (bottom horizontal scale) and pore diameter $d_{p}$ (top horizontal scale) in ordered porous $\mathrm{Si}$ samples with $\varphi=0.28$. Symbols are typically as large as the standard deviation of the calculated thermal conductivity. 
TABLE VI. Thermal conductivity $\kappa$ for various ordered porous Si samples with same porosity $\varphi=0.28$, but varying number of pores $N_{p}$ and pore diameter $d_{p}$.

\begin{tabular}{lcc}
\hline \hline$N_{p}$ & $d_{p}[\mathrm{~nm}]$ & $\kappa\left(\mathrm{W} \mathrm{m}^{-1} \mathrm{~K}^{-1}\right)$ \\
\hline 6 & 11.0 & 7.00 \\
28 & 6.6 & 1.75 \\
108 & 4.2 & 1.36 \\
224 & 3.3 & 0.78 \\
500 & 2.5 & 0.83 \\
1116 & 2.0 & 0.70 \\
\hline \hline
\end{tabular}

\section{Beyond the Eucken model}

All the present calculated AEMD data for whatever investigated system are in fact very accurately predicted by a model where the key structural parameter is the interface density $\Psi$. By generalizing Eq. (11), we can cast $\kappa_{\text {eff }}$ in the form

$$
\kappa_{\text {eff }}(\Psi)=\kappa_{\text {bulk }} \frac{1-d_{p} \Psi / 6}{1+d_{p} \Psi / 12+\Lambda_{\text {bulk }} \Psi / 4},
$$

where $\Psi$ now replaces all the structural parameters so far considered. We stress that although the interface density has been previously defined for spherical pores, it is actually independent from their shape and it can be used to identify universal trends in $k_{\text {eff }}$. We therefore argue that interface density, rather than the overall porosity, is a more effective quantity to fully describe thermal conductivity as a function of system conformation. As a matter of fact, samples with comparable interface density tend to have similar thermal conductivities, although having a different porosity. This is illustrated in Table VIII where systems with same porosity, but unlike interface density, show remarkably different thermal conductivity. On the other hand, when the interface density is similar, then so is thermal conductivity, regardless of the porosity.

In Fig. 12, we report $\kappa$ for the whole set of samples studied in this work (namely, any OPS and RPS) as a function of interface density (top) and porosity (bottom). The two curves are obtained by fitting the data using Eqs. (12) and (11) for $\Psi$

TABLE VII. Thermal conductivity $\kappa$ for various random porous Si samples with increasing porosity $\varphi$. Reported errors reflect a configurational average over four samples.

\begin{tabular}{cc}
\hline \hline$\varphi$ & $\kappa\left(\mathrm{W} \mathrm{m}^{-1} \mathrm{~K}^{-1}\right)$ \\
\hline 0.05 & $9.8 \pm 0.9$ \\
0.10 & $5.2 \pm 0.3$ \\
0.15 & $4.6 \pm 0.1$ \\
0.20 & $2.7 \pm 0.4$ \\
0.25 & $2.0 \pm 0.4$ \\
0.30 & $1.3 \pm 0.5$ \\
0.35 & $0.9 \pm 0.2$ \\
0.40 & $0.7 \pm 0.2$ \\
0.45 & $0.5 \pm 0.2$ \\
0.50 & $0.2 \pm 0.1$ \\
\hline
\end{tabular}

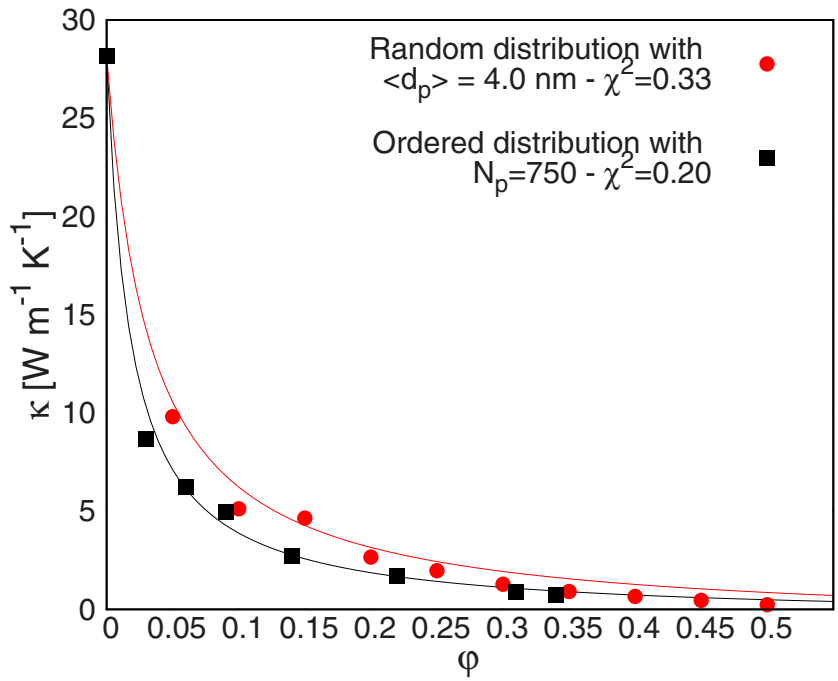

FIG. 11. (Color online) Thermal conductivity $\kappa$ as a function of porosity $\varphi$ in random (red dots) and ordered (black squares) porous $\mathrm{Si}$ samples. Solid lines: effective model provided by Eq. (11). Symbols are typically as large as the standard deviation of the calculated thermal conductivity.

and $\varphi$, respectively. The use of interface density, rather than porosity, as the main structural parameter in Eq. (12) gives rise to a more accurate fit of the data, as reflected by the reduced $\chi^{2}$ values, respectively: $\chi_{\Psi}^{2}=1.2$ and $\chi_{\varphi}^{2}=3.5$. The shaded regions in Fig. 12 top and bottom represent the deviation of the calculated AEMD data from the models provided by Eqs. (12) and (11), respectively. The smaller area of the red region stands for a better agreement, i.e., for the improved accuracy of the extended Eucken model provided by Eq. (12) based.

\section{CONCLUSIONS}

By nonequilibrium molecular dynamics simulations performed on systems large enough to faithfully describe both local scale and global structural features, we have investigated how thermal conductivity in ordered and random nanoporous silicon actually depends on porosity. In particular, we have thoroughly characterized the effects of pore size, shape and distribution on the resulting thermal transport properties. We have further used atomistic simulations to inform an extended version of the Eucken model, which is now valid both for macroporous and nanoporous systems.

TABLE VIII. Thermal conductivity $\kappa$ for three porous Si samples differing by interface density $\Psi$, pore diameter $d_{p}$, pore number $N_{p}$, and porosity $\varphi$.

\begin{tabular}{lcccc}
\hline \hline$\Psi\left(\mathrm{nm}^{-1}\right)$ & $d_{p}(\mathrm{~nm})$ & $N_{p}$ & $\varphi$ & $\kappa\left(\mathrm{W} \mathrm{m}^{-1} \mathrm{~K}^{-1}\right)$ \\
\hline 0.153 & 11.0 & 6 & 0.28 & 7.0 \\
0.840 & 2.0 & 1116 & 0.28 & 0.7 \\
0.164 & 3.3 & 72 & 0.09 & 6.2 \\
\hline \hline
\end{tabular}



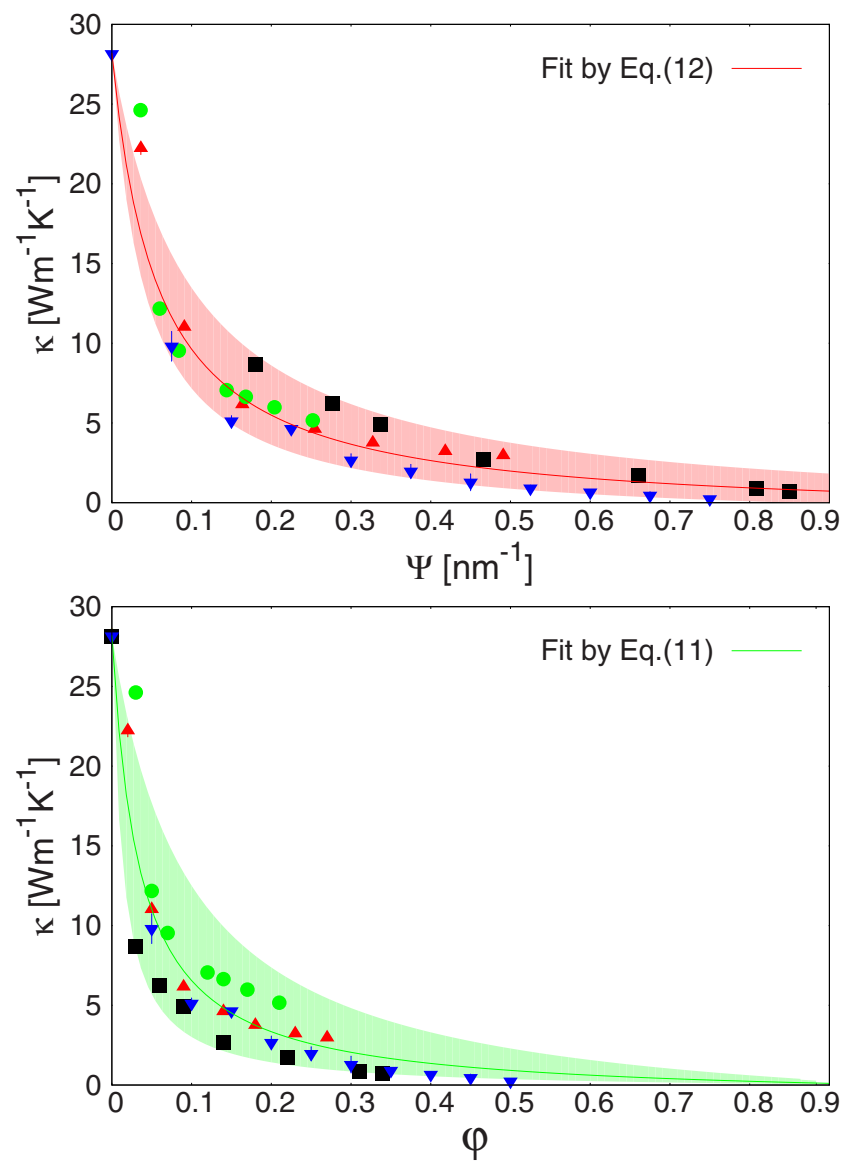

FIG. 12. (Color online) Symbols: Thermal conductivity $\kappa$ as function of the interface density $\Psi$ (top panel) and porosity $\varphi$ (bottom panel) for all systems here investigated. Solid lines: effective model provided by Eqs. (12) and (11) for the top and bottom panels, respectively. Shaded area: the deviation of the calculated AEMD data (symbols) from such models.
The main result of the present investigation is that the thermal conductivity $\kappa$ in nanoporous silicon does depend on porosity, as well as it is affected by the interface density $\Psi$, namely by the ratio between porosity and average pore diameter. Interestingly enough, $\Psi$ is identified as the real key structural characteristic of a nanoporous sample, fully determining its ability to transport heat.

A phenomenological effective model based on the interface density as the main variable has been accordingly elaborated, in accurate agreement with the present molecular dynamics results and fully describing the trends in thermal conductivity reduction due to the increase of the interface scattering in nanoporous systems. Such an effective model provides evidence that $\Psi$ is indeed a comparatively more effective parameter in describing trends in $\kappa$ than the overall sample porosity. The agreement between the present simulation data and the effective atomistic model here developed is very good, suggesting a possible way to measure the average size of the voids in a typical experimental nanoporous silicon sample. The present improved basic understanding of thermal transport properties in nanoporous silicon could allow to better tailor its structural features in order to meet possible applications as a key energy material, for instance, in thermoelectric production or thermal insulation.

\section{ACKNOWLEDGMENTS}

C.M. acknowledges Sardinia Regional Government for financial support (P.O.R. Sardegna ESF 2007-13). X.C. and R.R. acknowledge support by Ministerio de Economía y Competitividad (MINECO) under contracts Nos. FIS201237549-C05-05 and TEC2012-32305 (both co-funded by the EU under the FEDER program) and by the Generalitat de Catalunya under grants 2014 SGR 301 and 2014 SGR 384.
[1] Y. He, D. Donadio, J.-H. Lee, J. C. Grossman, and G. Galli, ACS Nano 5, 1839 (2011).

[2] Á. Miranda-Duràn, X. Cartoixà, M. C. Irisson, and R. Rurali, Nano Lett. 10, 3590 (2010).

[3] E. Garrone, F. Geobaldo, P. Rivolo, G. Amato, L. Boarino, M. Chiesa, E. Giamello, R. Gobetto, P. Ugliengo, and A. Viale, Adv. Mat. 5, 528 (2005).

[4] A. G. Nassiopoulou and G. Kaltsas, Phys. Stat. Sol. (A) 182, 307 (2000).

[5] J. H. Lee, J. C. Grossman, J. Reed, and G. A. Galli, Appl. Phys. Lett. 91, 223110 (2007).

[6] M. J. Sailor, Porous Silicon in Practice: Preparation, Characterization and Application (Wiley-VCH, Weinheim, Germany, 2012).

[7] A. Drost, P. Steiner, H. Moser, and W. Lang, Sensor. Mater. 7, 111 (1995).

[8] V. Bernini, P. Maddalena, E. Massera, and A. Ramaglia, Opt. Comm. 168, 305 (1995).

[9] G. Gesele, J. Linsmeier, V. Drach, J. Fricke, and R. ArensFischer, J. Phys. D: Appl. Phys. 30, 2911 (1997).
[10] J. Tang, H. T. Wang, D. H. Lee, M. Fardy, Z. Huo, T. P. Russell, and P. Yang, Nano Lett. 10, 4279 (2010).

[11] J. H. Lee, G. A. Galli, and J. C. Grossman, Nanolett. 8, 3750 (2008).

[12] A. Eucken, Ceram. Abstr. 11, 576 (1932).

[13] A. Eucken, Ceram. Abstr. 12, 231 (1933).

[14] E. Lampin, P. L. Palla, P. A. Francioso, and F. Cleri, J. Appl. Phys. 114, 033525 (2013).

[15] E. Lampin, Q.-H. Nguyen, P. A. Francioso, and F. Cleri, Appl. Phys. Lett. 100, 131906 (2012).

[16] C. Melis, R. Dettori, S. Vandermeulen, and L. Colombo, Eur. Phys. J. B 87, 96 (2014).

[17] C. Melis and L. Colombo, Phys. Rev. Lett. 112, 065901 (2014).

[18] K. R. Hahn, C. Melis, and L. Colombo, Eur. Phys. J. B 87, 150 (2014).

[19] S. Plimpton, J. Comp. Phys. 117, 1 (1995). See also the following site: http://lammps.sandia.gov.

[20] J. F. Justo, M. Z. Bazant, E. Kaxiras, V. V. Bulatov, and S. Yip, Phys. Rev. B 58, 2539 (1998). 
[21] D. P. Sellan and E. S. Landry and J. E. Turney and A. J. H. McGaughey and C. H. Amon, Phys. Rev. B. 81, 214305 (2010).

[22] Y. He, I. Savić, D. Donadio, and G. Galli, Phys. Chem. Chem. Phys. 14, 16209 (2012).

[23] A. S. Henry and G. Chem, J. Comp. Theo. Nano. 5, 1 (2008).

[24] F. Rodriguez-Reinoso, J. Rouquerol, K. K. Unger, and K. S. W. Sing, Characterization of Porous Solids III (Elsevier Science B.V., Amsterdam, Netherlands, 1994).

[25] P. K. Schelling, S. R. Phillpot, and P. Keblinski, Phys. Rev. B 65, 144306 (2002).

[26] P. E. Hopkins, P. T. Rakich, R. H. Olsson, I. F. El-Kady, and L. M. Phinney, Appl. Phys. Lett. 95, 161902 (2009).

[27] R. H. Tarkhanyan and D. G. Niarchos, APL Mater. 2, 076107 (2014).

[28] V. Jean, S. Fumeron, K. Termentzidis, S. Tutashkonko, and D. Lacroix, J. Appl. Phys. 115, 024304 (2014).
[29] D. Song and G. Chen, Appl. Phys. Lett. 84, 687 (2004).

[30] F. X. Alvarez, D. Jou, and A. Sellitto, Appl. Phys. Lett. 97, 033103 (2010).

[31] J. Fang and L. Pilon, J. Appl. Phys. 110, 064305 (2011).

[32] I. Sumirat, Y. Ando, and S. Shimamura, J. Porous Mater. 13, 439 (2006).

[33] A. Ould-Abbas, M. Bouchaour, and N.-E. C. Sari, Open J. Phys. Chem. 2, 1 (2012) [published online: http://www.SciRP.org/ journal/ojpc].

[34] L. Yang, Q. Zhai, H. Jiang, L. Han, J. Wang, and E. Wang, Chem. Commun. 49, 11415 (2013).

[35] S. Pèrichon, V. Lysenko, B. Remaki, D. Barbier, and B. Champagnon, J. Appl. Phys. 86, 4700 (1999).

[36] G. Benedetto, L. Boarino, and R. Spagnolo, Appl. Phys. A 64, 155 (1997). 